\title{
Diagnóstico clínico-radiológico de la neumonía del adulto adquirida en la comunidad
}

\author{
RODRIGO GIL D., PATRICIA FERNÁNDEZ V. y EDUARDO SABBAGH P.
}

\section{Clinical and radiological diagnosis of community-acquired pneumonia in adults}

Community acquired pneumonia in adults is an acute disease characterized by worsening in general conditions, fever, chills, cough, mucopurulent sputum and dyspnea; associated with tachycardia, tachypnea, fever and focal signs in pulmonary examination. The probability of pneumonia in a patient with acute respiratory symptoms depends on the disease prevalence in the environment where it is acquired and on clinical features. It is estimated that pneumonia prevalence is $3-5 \%$ in patients with respiratory disease seen in outpatient facilities. Clinical diagnosis of pneumonia without radiological confirmation lacks specificity because clinical presentation (history and physical examination) does not allow to differentiate pneumonia from other acute respiratory diseases (upper respiratory infections, bronchitis, influenza). Diagnosis must be based in clinical-radiological findings: clinical history and physical examination suggest the presence of pulmonary infection but accurate diagnosis is established when chest $\mathrm{X}$ ray confirms the existence of pulmonary infiltrates. Clinical findings and chest $\mathrm{X}$ ray do not permit to predict with certainty the etiology of pulmonary infection. Radiology is useful to confirm clinical suspicion, it establishes pneumonia location, its extension and severity; furthermore, it allows differentiation between pneumonia and other diseases, to detect possible complications, and may be useful in follow up of high risk patients. The resolution of radiological infiltrates often ensues several weeks or months after clinical recovery, especially in the elderly and in multilobar pneumonia cared for in intensive care units.

Key words: pneumonia, diagnosis, symptoms, signs, radiography.

Palabras clave: neumonía, diagnóstico, sintomas, signos, radiología.

\section{Presentación clínica de la neumonía adquirida en la comunidad}

Clásicamente la neumonía adquirida en la comunidad (NAC) del adulto se define como un cuadro de evolución aguda, caracterizado por compromiso del estado general, fiebre, calofríos, tos, expectoración purulenta y dificultad respiratoria de magnitud variable; asociado en el examen físico a taquicardia ( $>100$ latidos/min), taquipnea ( $>20$ respiraciones/min), fiebre $\left(>37,8^{\circ} \mathrm{C}\right)$, y signos focales en el examen pulmonar: matidez, disminución del murmullo pulmonar, crepitaciones, broncofonía y egofonía. Sin embargo, esta descripción no ha sido plan- teada en términos de probabilidad, que suele ser la realidad de la práctica clínica, en la que debemos definir si iniciamos tratamiento antibiótico con los elementos clínicos entregados por la historia y examen físico, o debemos solicitar exámenes de laboratorio complementarios. Esta decisión no es trivial ya que la neumonía es la única infección respiratoria aguda en que un retardo en el inicio del tratamiento antibiótico se ha asociado a un mayor riesgo de complicaciones y muerte ${ }^{1}[\mathbf{I I}]$. En varios estudios se ha comunicado que la probabilidad de un paciente con síntomas respiratorios agudos de tener una neumonía depende de la prevalencia de la enfermedad en el ambiente donde se presenta y de

Instituto Nacional del Tórax, Santiago, Chile, 
las manifestaciones clínicas del enfermo ${ }^{2-4}$. Es por ello que diversos estudios han comunicado tasas de prevalencia de neumonía muy variables, oscilando entre 2,6 y $46 \% \%^{2,3}$. En general, se estima que la prevalencia de neumonía en los servicios de atención ambulatoria (consultorios y servicios de urgencia) corresponde a 3-5\% de las consultas respiratorias ${ }^{5}$. La prevalencia aumenta en la población senescente con comorbilidad múltiple y pobre acceso a los servicios de atención médica, y disminuye en los pacientes jóvenes, sin comorbilidad y con buen acceso a la atención médica. Si estimamos una prevalencia de 5\% de neumonía en los pacientes que consultan por tos en los servicios de atención primaria, el paciente que consulta por tos y fiebre tendría una probabilidad de neumonía de alrededor de $20 \%$, si tuviera tos y crepitaciones en el examen pulmonar la probabilidad sería entre 8 y $10 \%$, y si tuviera matidez torácica la probabilidad oscilaría entre 10 y $18 \%{ }^{6}$. De este modo, es posible afirmar que ninguno de los síntomas o signos, analizados aisladamente, aumentan en forma significativa la probabilidad de un paciente de tener neumonía ${ }^{6}$ [Ia]. Tampoco la ausencia de cualquiera de los signos clásicos reduce en forma significativa la probabilidad de neumonía ${ }^{7}$. El problema diagnóstico se agrava cuando se considera que existe una gran variabilidad en la pesquisa de signos físicos anormales en el examen pulmonar entre distintos examinadores $^{4,8}$ [II] . Sin embargo, en la práctica clínica los médicos analizan los síntomas y signos en forma conjunta para definir un diagnóstico. Cuando se analizan grupos de síntomas y signos, la capacidad diagnóstica mejora sólo parcialmente; por ejemplo si un paciente consulta por tos y tiene fiebre, taquicardia y crepitaciones la probabilidad que tenga una neumonía varía entre 18 y $42 \%$. Si el escenario incluye pacientes graves, como ocurre en los servicios de urgencia, la prevalencia aumenta de 5 a $10 \%$, con lo que la probabilidad de neumonía con los mismos síntomas y signos aumenta entre 32 y $60 \%{ }^{6}$. Se han diseñado varias fórmulas que intentan predecir la probabilidad de neumonía en un determinado paciente ${ }^{9}$ [II] ; sin embargo, habitualmente se requiere una radiografía de tórax para confirmar el diagnóstico. Uno de estos modelos sugiere que un paciente que consulta por fiebre $\left(>37,8^{\circ} \mathrm{C}\right)$, tos, expectoración, taquipnea (> 25 respiraciones/min), mialgias y sudoración nocturna, en ausencia de odinofagia y rinorrea, tendría con alta probabilidad una NAC ${ }^{2}$. Este modelo predictor clínico tiene una sensibilidad de $91 \%$ y una especificidad de $40 \%$. Por el contrario, un estudio realizado en un servicio de urgencia sugiere que si un paciente consulta por tos y los signos vitales son normales (es decir, no tiene fiebre, taquicardia ni taquipnea), la probabilidad que tenga neumonía disminuye significativamente ${ }^{7}$.

Basados en estos antecedentes, la historia y examen físico carecen de sensibilidad y especificidad para establecer el diagnóstico clínico, la Sociedad Americana de Tórax (American Thoracic Society-ATS ${ }^{10}$ y la Sociedad Americana de Enfermedades Infecciosas (Infectious Diseases Society of AmericaIDSA) ${ }^{11}$ han recomendado que se debe solicitar radiografía de tórax a todos los pacientes con sospecha clínica de neumonía. Por otra parte, la Sociedad Británica de Tórax (British Thoracic Society-BTS) ${ }^{9}$ no recomienda el uso rutinario de radiografía de tórax en el manejo ambulatorio de pacientes con neumonía considerando las características del sistema de salud del Reino Unido. En la guía clínica de la BTS ${ }^{9}$ se define la NAC empleando criterios clínicos: historia sugerente de una infección respiratoria baja aguda (tos asociada a uno o más síntomas de infección respiratoria baja); presencia de signos focales en el examen del tórax; la presencia al menos de uno de los siguientes síntomas y signos: fiebre, calofríos, sudoración, mialgias, o temperatura igual o superior a $38^{\circ} \mathrm{C}$; ausencia de otra explicación para la enfermedad respiratoria aguda.

\section{El cuadro clínico ¿permite diferenciar con certeza el paciente con neumonía adquirida en la comunidad de otras condiciones respi- ratorias agudas?}

El diagnóstico clínico de neumonía sin una radiografía de tórax carece de precisión ya que el cuadro clínico (historia y examen físico) no permite diferenciar con certeza al paciente con neumonía de otras condiciones respiratorias agudas ${ }^{12}$ [Ia]. El diagnóstico de NAC basado exclusivamente en criterios clínicos se ve dificultado por la gran variabilidad que existe en la detección de signos focales en el examen de tórax entre distintos observadores ${ }^{4,8}$ [Ia]. Sin embargo, en el medio ambulatorio a los pacientes con síntomas respiratorios agudos que no presentan anomalías en el estado de conciencia y los signos vitales, el diagnóstico de neumonía resulta muy improbable, así en este contexto clínico no se recomendaría solicitar una radiografía de tó$\operatorname{rax}^{12}[\mathbf{A +}]$. 


\section{Dificultades en el diagnóstico clínico de neumonía en categorías específicas: adulto mayor y portadores de enfermedad cardiopulmonar crónica}

El adulto mayor ( $>65$ años) suele tener neumonías de presentación atípica que dificultan el diagnóstico y retrasan el inicio de tratamiento, afectando adversamente el pronóstico de los enfermos ${ }^{13-15}$. Estos pacientes suelen no presentar los síntomas respiratorios clásicos y/o fiebre, consultando por síntomas inespecíficos como decaimiento, anorexia, estado mental alterado, o por descompensación de enfermedades crónicas $^{13,14}$ [II]. Por otra parte, los adultos mayores frecuentemente tienen comorbilidades como insuficiencia cardíaca congestiva, diabetes mellitus, insuficiencia renal crónica y enfermedad pulmonar obstructiva crónica (EPOC), cuya sintomatología dificulta aún más la pesquisa de los signos clínicos clásicos de la neumonía.

En suma, el diagnóstico de neumonía se debe plantear frente a un paciente que se presenta con tos y compromiso del estado general, especialmente si la tos se acompaña de expectoración mucopurulenta y fiebre. Los signos clásicos de neumonía como la matidez torácica, crepitaciones y respiración soplante son útiles cuando están presentes, pero no son sensibles en el diagnóstico de NAC. Los pacientes de edad avanzada con NAC pueden no tener los síntomas clásicos de la infección respiratoria aguda, y en cambio consultan por alteración del nivel de conciencia, rechazo alimentario o descompensación de sus enfermedades crónicas [II]. La radiografía de tórax sigue siendo el examen de referencia para el diagnóstico de neumonía [II].

\section{Recomendaciones}

Sobre la base de las evidencias disponibles, considerando que la historia y examen físico no permiten diferenciar con certeza la neumonía de otras afecciones respiratorias agudas, la Sociedad Chilena de Enfermedades Respiratorias recomienda que la confirmación del diagnóstico de NAC se haga con una radiografía de tórax [B]. Como una forma de racionalizar el uso de este examen en el medio ambulatorio, logrando una mejor relación costo beneficio, recomendamos solicitar examen radiográfico en las siguientes circunstancias clínicas [B]:

- Paciente adulto que consulta por tos, expectoración, fiebre y/o dificultad respiratoria aguda, y presenta algún signo focal en el examen pulmonar.

- Paciente mayor de 65 años con compromiso de conciencia y/o descompensación de una enfermedad crónica de causa desconocida.

- Paciente con insuficiencia cardíaca, EPOC u otra enfermedad pulmonar crónica, que consulta por tos, expectoración o fiebre, independientemente de los hallazgos en el examen pulmonar.

Por otra parte, si un paciente consulta por tos y/o expectoración y no tiene alteraciones en los signos vitales y en el estado de conciencia, sugerimos no solicitar una radiografía de tórax [B]. La probabilidad de neumonía disminuye significativamente en los pacientes que consultan por tos y/o fiebre con menos de 48 horas de evolución asociado a odinofagia, rinorrea y ausencia de signos focales en el examen pulmonar; por lo tanto, a pacientes en esta categoría se recomienda no solicitar radiografía de tórax [B]. Por último, si existe la sospecha clínica de NAC, y el médico clínico no dispone de una radiografía de tórax, se recomienda iniciar el tratamiento antibiótico lo antes posible, porque su retraso aumenta el riesgo de complicaciones y muerte [B].

\section{Utilidad del cuadro clínico-radiográfico en el diagnóstico de la neumonía adquirida en la comunidad típica y atípica}

Durante largo tiempo, los médicos clínicos han diferenciado el cuadro clínico de la NAC clásica o "típica" y la neumonía "atípica". Se pensaba que el cuadro clínico de la neumonía típica era ocasionado por patógenos respiratorios clásicos como Streptococcus pneumoniae o Haemophilus influenzae, y se caracterizaba por síntomas respiratorios agudos: compromiso del estado general, tos, expectoración purulenta, fiebre, calofríos, dolor torácico, y signos de consolidación en el examen pulmonar, asociado a leucocitosis y desviación a izquierda en el hemograma, y un foco de condensación lobar en la radiografía de tórax. Por otra parte, se consideraba que las neumonías atípicas eran producidas por otros microorganismos como Mycoplasma pneumoniae, Chlamydia pneumoniae y Legionella pneumophila, que se manifestaban por síntomas respiratorios de evolución subaguda, con escaso compromiso del estado general, febrículas, ausencia de expectoración y escasos signos focales en el examen del tórax, y la presencia de infiltrados intersticiales en la radiografía de tórax. Sin embargo, estudios recientes han demostrado que no existen síntomas y signos clínicos ni radiográficos de pre- 
sentación de una NAC que permitan predecir con certeza el agente patógeno que la ocasionó ${ }^{16,17}$ [II] $]$.

\section{Recomendación}

El término neumonía atípica debiera abandonarse ya que sugiere incorrectamente que existe una presentación clínica característica de las neumonías producidas por microorganismos específicos (M. pneumoniae, C. pneumoniae, L. pneumophila $)^{9}$ [B]. Sin embargo, ha sido útil denominar a estos microorganismos como atípicos ya que en general requieren de una cobertura antibiótica común. El cuadro clínico (historia y examen físico) y los hallazgos de la radiografía de tórax no permiten predecir con certeza el agente etiológico de la infección pulmonar [B].

\section{Descripción del cuadro radiológico de la neumonía del adulto adquirida en la comunidad}

La radiografía de tórax frontal y lateral debe ser uno de los exámenes de rutina en el diagnóstico y evaluación de los pacientes con NAC. Permite confirmar el diagnóstico clínico y establecer su localización, extensión y gravedad, además permite diferenciar la neumonía de otras patologías, detectar posibles complicaciones, y puede ser útil en el seguimiento de los pacientes de alto riesgo [II, III]. Ocasionalmente nos servirá para evaluar patologías concomitantes que pueden estar facilitando la infección y/o modificando su evolución, como procesos obstructivos bronquiales neoplásicos o de otro tipo, enfisema pulmonar, fibrosis, bronquiectasias, etc. En este último aspecto, en muchos casos será necesario complementar el estudio con una tomografía computada de tórax, pero siempre orientado por la sospecha clínica y los hallazgos de la radiografía de tórax. En el medio ambulatorio, se recomienda solicitar una radiografía de tórax a los pacientes con cuadro clínico sugerente de neumonía para certificar el diagnóstico y evaluar la gravedad del cuadro [B].

\section{La radiografía de tórax ¿permite diferenciar con certeza el paciente con neumonía adquirida en la comunidad de otras condiciones respiratorias agudas?}

El diagnóstico diferencial de una NAC en el adulto inmunocompetente incluye una gran variedad de otras condiciones patológicas, y no es posible abordarlas en forma extensa en esta guía (Tabla 1). Sin embargo, en nuestro país la tu- berculosis es uno de los diagnósticos diferenciales que se debe plantear en todo paciente que consulta por tos y expectoración persistente durante dos semanas, a quienes se recomienda solicitar dos baciloscopias de expectoración, y una muestra de expectoración para cultivo de Koch [D]. Los hallazgos de la radiografía de tórax son inespecíficos y pueden ser ocasionados por múltiples enfermedades infecciosas y no infecciosas que afectan el parénquima pulmonar [II]. De este modo, el diagnóstico de neumonía debe estar sustentado en un cuadro clínico compatible (fiebre, tos, expectoración, calofríos, dolor torácico, disnea) asociado a la presencia de infiltrados radiográficos de aparición reciente [B].

\section{El cuadro radiográfico ¿permite predecir la etiología de la neumonía?}

No se ha demostrado que existan patrones radiológicos característicos que permitan predecir la etiología de la neumonía ${ }^{18-20}$ [II]. Sin embargo, algunos estudios sugieren que se pueden establecer algunas diferencias, por ejemplo, las neumonías con imagen de consolidación homogénea son menos frecuentes en infecciones por M. pneumoniae, C. pneumoniae y virus respiratorios. Por otra parte, la neumonía multilobar y la presencia de efusión pleural son más frecuentes en la infección neumocócica bacteriémica ${ }^{18}$

Tabla 1. Diagnóstico diferencial de la neumonía del adulto adquirida en la comunidad

Tuberculosis pulmonar

Insuficiencia cardíaca congestiva

Tromboembolismo pulmonar

Atelectasia pulmonar

Neumonitis por hipersensibilidad

Daño pulmonar por fármacos

Daño pulmonar por radioterapia

Enfermedad pulmonar neoplásica primaria y metastásica

Linfangiosis carcinomatosa

Enfermedad colágeno-vascular: lupus eritematoso sistémico, artritis reumatoidea, granulomatosis de Wegener, síndrome de Churg-Strauss.

Sarcoidosis

Neumonía eosinofílica

Neumonía en organización criptogénica 
[III]. En la neumonía estafilocócica es más frecuente el compromiso radiográfico multilobar, la cavitación, los neumatoceles, y el neumotórax espontáneo [III]. En la neumonía por Klebsiella pneumoniae es frecuente el compromiso de los lóbulos superiores y el abombamiento de la cisura interlobar; sin embargo, ambos hallazgos son inespecíficos y pueden ser observados en la neumonía neumocócica y por Staphylococcus aureus [II]. En varios estudios no se han demostrado diferencias significativas entre los patrones radiológicos ocasionados por los microorganismos típicos y atípicos ${ }^{19,20}$ [III].

No se recomienda emplear criterios radiológicos para intentar predecir el agente causal de la infección pulmonar y orientar el tratamiento antibiótico empírico [B].

\section{¿Cuál es la velocidad de resolución de los infiltrados radiográficos en la neumonía del adulto adquirida en la comunidad?}

La resolución de los infiltrados radiográficos suele ser más lenta que la mejoría clínica de los enfermos. La resolución completa de las alteraciones radiológicas ocurre en alrededor del 50\% de los pacientes a las dos semanas de evolución, $64 \%$ a las cuatro semanas y $73 \%$ a las seis semanas ${ }^{21}[\mathbf{I I}]$. La resolución es más lenta en la neumonía neumocócica bacteriémica y por Legionella sp [III]. También la resolución suele ser más lenta en los adultos mayores, en los portadores de enfermedad cardiopulmonar crónica, en la neumonía multilobar y en los pacientes admitidos al hospital. Por el contrario, la mejoría radiológica de la infección por microorganismos atípicos (M. pneumoniae, C. pneumoniae, virus respiratorios) suele ser más rápida que la ocasionada por agentes clásicos [III].

En pacientes con evolución clínica favorable, no se recomienda realizar estudios complementarios sólo porque los infiltrados en la radiografía de tórax se demoran en mejorar [B].

\section{¿Cuándo debe repetirse la radiografía de tórax y qué debe hacerse si no se ha normalizado?}

No existen evidencias concluyentes que permitan dar una recomendación respecto de esta pregunta. En la práctica clínica se suele solicitar una radiografía de control previo al alta en los pacientes hospitalizados por NAC o en la visita de seguimiento a las 4 a 6 semanas. Sin embargo, no existen evidencias que avalen esta recomendación en los pacientes que evolucionan fa- vorablemente de la infección pulmonar [III]. En general, si el paciente evoluciona bien desde el punto de vista clínico, no parece necesario hacerlo antes de 4 a 6 semanas. El motivo principal de hacer un control radiológico es diagnosticar un cáncer pulmonar que se hubiera complicado con una neumonía o la pesquisa de otra patología infecciosa o no infecciosa (tuberculosis, neumonitis criptogénica en organización (cryptogenic organizing pneumonitis- COP), daño pulmonar por fármacos, etc).

La radiografía de tórax no necesita ser repetida antes del egreso en los pacientes hospitalizados por neumonía que evolucionan satisfactoriamente [D]. Se recomienda repetir la radiografía de tórax buscando un cáncer pulmonar en todos los pacientes fumadores mayores de 60 años que hayan tenido una neumonía [B]. Además, se recomienda solicitar una radiografía de tórax de control a todos los pacientes con síntomas y signos respiratorios persistentes después de 6 a 8 semanas de evolución [C].

\section{Resumen}

La neumonía del adulto adquirida en la comunidad es un cuadro de evolución aguda, caracterizado por compromiso del estado general, fiebre, calofríos, tos, expectoración mucopurulenta y dificultad respiratoria; asociado en el examen físico a taquicardia, taquipnea, fiebre y signos focales en el examen pulmonar. La probabilidad que un paciente con síntomas respiratorios agudos tenga una neumonía depende de la prevalencia de la enfermedad en el ambiente donde se presenta y de las manifestaciones clínicas del enfermo. Se estima que la prevalencia de neumonía en los servicios de atención ambulatoria corresponde a 3-5\% de las consultas por patología respiratoria. El diagnóstico clínico de neumonía sin confirmación radiográfica carece de precisión ya que el cuadro clínico (historia y examen físico) no permite diferenciar con certeza al paciente con neumonía de otras condiciones respiratorias agudas (infecciones de la vía aérea superior, bronquitis, influenza). El diagnóstico de neumonía es clínico-radiográfico: la historia y examen físico sugieren la presencia de una infección pulmonar, pero el diagnóstico de certeza se establece cuando se confirma la presencia de infiltrados pulmonares en la radiografía de tórax. El cuadro clínico y los hallazgos de la radiografía de tórax no permiten predecir con certeza el agente etiológico de la infección pulmonar. La radiografía de tórax permite confirmar el diagnóstico clínico, establecer su localización, extensión y gravedad; además permite diferenciar la neumonía de otras patologías, detectar posibles complicaciones, y puede ser útil en el seguimiento de los pacientes de alto riesgo. La resolución de los infiltrados radiográficos a menudo 
ocurre varias semanas o meses después de la mejoría clínica, especialmente en el anciano y en la neumonía multilobar manejada en la UCI.

\section{Bibliografía}

1.- Meehan T P, Fine M J, Krumholz H M, Scinto J D, Galusha D H, Mockalis J T, et al. Quality of care, process, and outcomes in elderly patients with pneumonia. JAMA 1997; 278: 2080-4.

2.- Diehr P, Wood R W, Bushyhead J, Krueger L, Wolcott $\mathrm{B}$, Tompkins $\mathrm{R} \mathrm{K}$. Prediction of pneumonia in outpatients with acute cough, a statistical approach. J Chronic Dis 1984; 37: 215-25.

3.- Heckerling P S, Tape T G, Wigton R S, Hissong K K, Leikin J B, Ornato J P, et al. Clinical prediction rule for pulmonary infiltrates. Ann Intern Med 1990; 113: 664-70.

4.- Wipf J E, Lipsky B A, Hirschmann J V, Boyko E J, Takasugi J, Peugeot R L, et al. Diagnosing pneumonia by physical examination: relevant or relic? Arch Intern Med 1999; 159: 1082-7.

5.- Metlay J P, Stafford R S, Singer D E. National trends in the use of antibiotics by primary care physicians for adult patients with cough. Arch Intern Med 1998; 158: 1813-8.

6.- Metlay J P, Fine M J. Testing strategies in the initial management of patients with community-acquired pneumonia. Ann Intern Med 2003; 138: 109-18.

7.- Gennis P, Gallagher J, Falvo C, Baker S, Than W. Clinical criteria for the detection of pneumonia in adults: guidelines for ordering chest roentgenograms in the emergency department. J Emerg Med 1989; 7: 263-8.

8.- Spireti M A, Cook D G, Clarke S W. Reliability of eliciting physical signs in examination of the chest. Lancet 1988; 1: 873-5.

9.- British Thoracic Society Standards of Care Committee. BTS Guidelines for the Management of Community Acquired Pneumonia in Adults. Thorax 2001; 56 (Suppl 4): iv1-iv64.

10.- Niederman M S, Mandell L A, Anzueto A, Bass J B, Broughton W A, Campbell G D, et al. Guidelines for the management of adults with community-acquired pneumonia. Diagnosis, assessment of severity, antimicrobial therapy, and prevention. Am J Respir Crit Care Med 2001; 163: 1730-54.
11.- Bartlett J G, Dowell S F, Mandell L A, File Jr T M, Musher D M, Fine M J. Practice guidelines for the management of community-acquired pneumonia in adults. Infectious Diseases Society of America. Clin Infect Dis 2000; 31: 347-82.

12.- Metlay J P, Kapoor W N, Fine M J. Does this patient have community-acquired pneumonia? Diagnosing pneumonia by history and physical examination. JAMA 1997; 278: 1440-5.

13.- Marrie T J. Pneumonia in the elderly. Curr Opin Pulm Med 1996; 2: 192-7.

14.- Riquelme R, Torres A, El-Ebiary M, Mensa J, Estruch $\mathrm{R}$, Ruíz $\mathrm{M}$, et al. Community-acquired pneumonia in the elderly. Clinical and nutritional aspects. Am J Respir Crit Care Med 1997; 156: 1908-14.

15.- Saldías F, O`brien A, Gederlini A, Farías G, Díaz A. Neumonía adquirida en la comunidad en el anciano inmunocompetente que requiere hospitalización. Cuadro clínico, factores pronósticos y tratamiento. Arch Bronconeumol 2003; 39: 333-40.

16.- Fang G D, Fine M, Orloff J, Arisumi D, Yu V L, Kapoor W, et al. New and emerging etiologies for community-acquired pneumonia with implications for therapy. A prospective multicenter study of $359 \mathrm{ca}-$ ses. Medicine 1990; 69: 307-16.

17.- Farr B M, Kaiser D L, Harrison B D, Connolly C K. Prediction of microbial aetiology at admission to hospital for pneumonia from the presenting clinical features. British Thoracic Society Pneumonia Research Subcommittee. Thorax 1989; 44: 1031-5.

18.- Macfarlane J T, Miller A C, Roderick Smith W H, Morris A H, Rose D H. Comparative radiographic features of community acquired Legionnaires' disease, pneumococcal pneumonia, mycoplasma pneumonia, and psittacosis. Thorax 1984; 39: 28-33.

19.- Lieberman D, Ben-Yaakov M, Lazarovich Z, Porath A, Schlaeffer F, Lieberman D, et al. Chlamydia pneumoniae community-acquired pneumonia: a review of 62 hospitalized adult patients. Infection 1996; 24 : 109-14.

20.- Lieberman D, Porath A, Schlaeffer F, Lieberman D, Boldur I. Legionella species community-acquired pneumonia. A review of 56 hospitalized adult patients. Chest 1996; 109: 1243-9.

21.- Mittl R L Jr, Schwab R J, Duchin J S, Goin J E, Albeida S M, Miller W T. Radiographic resolution of community-acquired pneumonia. Am J Resp Crit Care Med 1994; 149 (3 Pt 1): 630-5.

Correspondencia a:

Rodrigo Gil D.

rodrigo.gil@terra.cl 